\title{
Cytometric fingerprints: evaluation of new tools for analyzing microbial community dynamics
}

\author{
Christin Koch ${ }^{1}$, Falk Harnisch ${ }^{1,2}{ }^{*}$, Uwe Schröder $^{2}$ and Susann Müller ${ }^{1}$ \\ ${ }^{1}$ Department of Environmental Microbiology, UFZ - Helmholtz-Centre for Environmental Research, Leipzig, Germany \\ 2 Institute of Environmental and Sustainable Chemistry, TU Braunschweig, Braunschweig, Germany
}

\section{Edited by:}

Frederik Hammes, Eawag, Swiss

Federal Institute of Aquatic Science and Technology, Switzerland

\section{Reviewed by:}

Raffaella Balestrini, Consiglio

Nazionale delle Ricerche, Italy

Stefano Amalfitano, Istituto di

Ricerca Sulle Acque (IRSA-CNR),

Italy

*Correspondence:

Falk Harnisch, Department of Environmental Microbiology, UFZ -

Helmholtz Centre for Environmental

Research, Permoserstr. 15,

04318 Leipzig, Germany

e-mail: falk.harnisch@ufz.de
Optical characteristics of individual bacterial cells of natural communities can be measured with flow cytometry (FCM) in high throughput. The resulting data are visualized in cytometric histograms. These histograms represent individual cytometric fingerprints of microbial communities, e.g., at certain time points or microenvironmental conditions. Up to now four tools for analyzing the variation in these cytometric fingerprints are available but have not yet been systematically compared regarding application: Dalmatian Plot, Cytometric Histogram Image Comparison (CHIC), Cytometric Barcoding (CyBar), and FlowFP. In this article these tools were evaluated concerning (i) the required experience of the operator in handling cytometric data sets, (ii) the detection level of changes, (iii) time demand for analysis, and (iv) software requirements. As an illustrative example, FCM was used to characterize the microbial community structure of electroactive microbial biofilms. Their cytometric fingerprints were determined, analyzed with all four tools, and correlated to experimental and functional parameters. The source of inoculum (four different types of wastewater samples) showed the strongest influence on the microbial community structure and biofilm performance while the choice of substrate (acetate or lactate) had no significant effect in the present study. All four evaluation tools were found suitable to monitor structural changes of natural microbial communities. The Dalmatian Plot was shown to be most sensitive to operator impact but nevertheless provided an overview on community shifts. CHIC, CyBar, and FlowFP showed less operator dependence and gave highly resolved information on community structure variation on different detection levels. In conclusion, experimental and productivity parameters correlated with the biofilm structures and practical process integration details were available from cytometric fingerprint analysis.

Keywords: microbial flow cytometry, cytometric fingerprinting, microbial fuel cells, electrochemical active microbial biofilms, natural communities, cytometric data analysis, cytometric pattern analysis

\section{INTRODUCTION}

Flow cytometry (FCM) is a high throughput method for analysis of optical characteristics of cells. Its main advantages are the fast analysis, high measuring accuracy, and sensitivity on the single cell level. Thus, FCM is widely applied for biological analysis, especially in medical routine diagnosis and medical research. Compared to that, the application of FCM for the characterization of microbial cells is less common (Web of Knowledge "flow cytometry" 85,039 hits, "flow cytometry bacteria" 3093 hits, 2014/03/17). Especially the characterization of complex microbial communities by FCM, which is termed cytometric fingerprinting, is still rare. Therefore, the following article compares and discusses recently published methods for FCM data analyses of complex microbial communities to expand the application of cytometric fingerprinting.

The measuring principle of FCM is the following (see also Figure 1): the individual cells of a microbial community are arranged within a liquid stream by hydrodynamic focusing. The cells individually pass a laser beam and thereby their intrinsic properties (cell size, morphology, and granularity) lead to specific interactions with the laser light, including light scattering and fluorescence. Usually, the scattered and refracted light is detected at low angle, i.e., below $2^{\circ}$ deviation from the incident light beam that is denominated as Forward Scatter (FSC), or perpendicular to the incident light, denominated as Sideward Scatter (SSC) (Shapiro, 2003) (Figure 1B). Furthermore, fluorescence light can be detected if suitable fluorophores are present. Thus, every cell is represented by an individual set of optical parameters and the acquisition of this set of parameters is performed for every single cell during FCM measurement. In a two dimensional (2D) histogram all cells of a given sample are represented as virtual cells (see Box 1) visualizing the cell's characteristics regarding the chosen optical parameters. Consequently, if cells have very similar optical characteristics clusters of virtual cells will be created in a histogram. These clusters represent subcommunities of a microbial community and thus are of high interest for data analysis, as demonstrated below.

Cytometric fingerprinting can be used to detect changes in the structure of microbial communities. If cells disappear or accumulate virtually in certain positions of a $2 \mathrm{D}$-histogramm or alter their optical characteristics these structural community changes become visible by comparing the cytometric fingerprints of two 


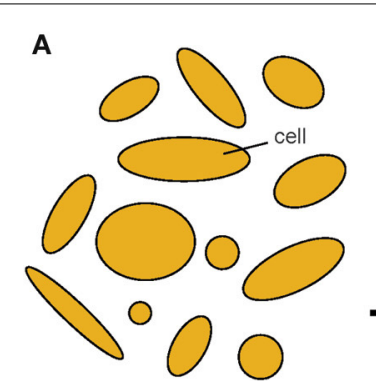

Microbial community

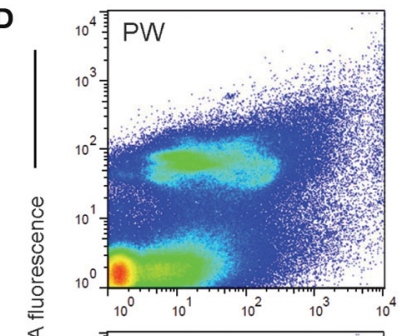

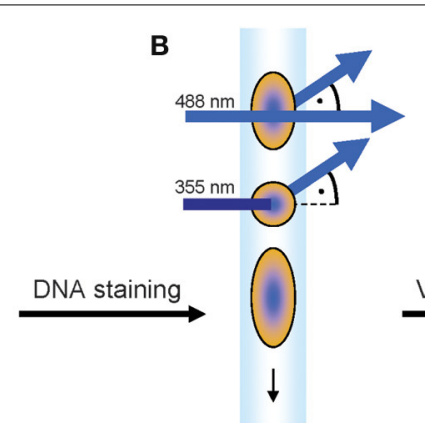

FC analysis

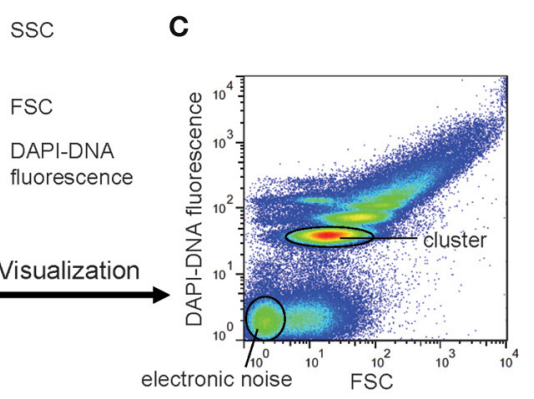

Cytometric 2D histogram
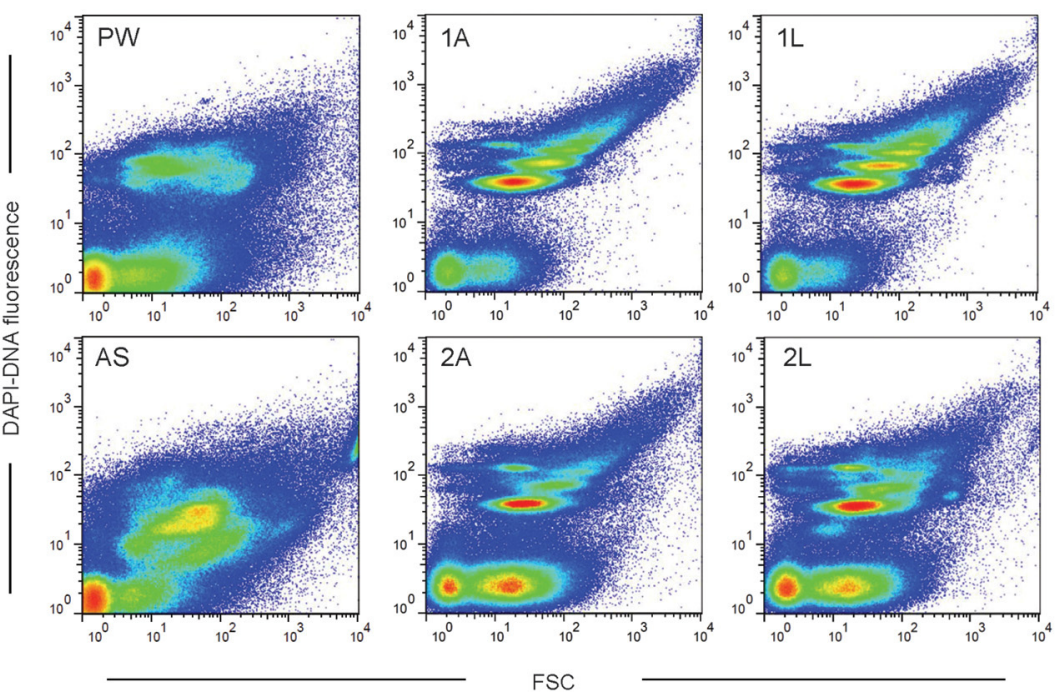

FIGURE 1| Natural microbial communities comprise a high diversity of uncharacterized microbial cells (A). The individual structure of these microbial communities can be characterized using FCM. The cells are stained with the DNA specific binding molecule DAPI and the cellular characteristics FSC and DAPI-DNA fluorescence can be recorded (B). The cytometric histogram visualizes the measurement of

one sample (C). Each virtual cell in the histogram represents the characteristics of a cell regarding the two chosen optical parameters. Therewith, the cytometric histogram can be regarded as cytometric fingerprint of the microbial community structure. Different microbial communities will be characterized by differences in their cytometric fingerprints (D). sampling points. A typical measurement for characterizing natural microbial communities includes 250,000 cells and takes about $3 \mathrm{~min}$. Here, FSC and fluorescence are established parameters for characterization (Kleinsteuber et al., 2006; Günther et al., 2009). Thus, as usually most cells show no substantial autofluorescence, it is necessary to use a fluorescence staining labeling all microbial cells. As every microbial cell contains DNA, the use of the highly specific DNA-binding molecule 4',6-diamidino-2-phenylindole (DAPI) is recommended (Meistrich et al., 1978). However, it should be considered that the effectivity of staining can vary, depending, e.g., on the cell type and state (Müller and Nebe-vonCaron, 2010). When using DAPI not only all cells are stained (and thus can be detected), but also their cellular DNA content can be quantified by the fluorescence intensity. As the cellular DNA content is dependent on cell proliferation and cell division states, environmental alterations causing variations in growth velocity and, therewith, in proliferation activity can easily be detected using FCM (Müller, 2007). A variation of other detectable intrinsic cell properties, e.g., the cell size related distribution, is reflected by the FSC signal. Therefore, the resulting cytometric fingerprint based on FSC and the DAPI-DNA fluorescence in a 2D histogram (Figure 1C) represents the microbial community structure at the point of measurement. This fingerprint is (almost) unique by the number of cell clusters, the position of these clusters in the histogram, and the numbers of cells within each cluster (Koch et al., 2013c).

When looking at various FCM histograms, i.e., respective cytometric fingerprints, differences can be spotted by the naked eye (e.g., Koch et al., 2013c). However, the challenge is to quantify these differences. Therefore, the information of two parameters for each virtual cell has to be transferred to a matrix that is suitable for evaluation. Only then FCM histograms can be exploited for further analysis, e.g., for following dynamics of microbial community structures in response to environmental changes or to compare microbial communities from different origins.

Four methods have recently been applied to analyze changes in the microbial community structure based on cytometric fingerprint measurements: Dalmatian Plot (Bombach et al., 2011), 


\section{Box 1 | Definitions.}

Microbial community: Is the entity of microorganism in a natural sample. It can comprise high diversity, i.e., hundreds of different species regarding phylogeny and function.

Cell: The microbial cell is an individual biological unit. It is characterized by optical properties which can be measured using FCM.

Virtual cell: The virtual cell represents the cell's characteristics regarding the chosen optical parameters usually in a 2D histogram.

Cluster: Virtual cells with similar optical properties. In microbial community analysis a cluster is representative of a microbial subcommunity.

Segregated data analysis: Allows a differentiated (or discriminated) analysis of cytometric data sets. It is possible with gate or grid information (see below).

Gate: A gate marks a cluster of cells in the histogram that differ from others in their optical properties. It can be defined using one, two, or even more parameters. Methods in microbiology: Dalmatian Plot, CyBar.

Grid: The use of a geometrical grid is an alternative to cluster based gating of FCM histograms. Methods in microbiology: Quadrant markers, FlowFP. Image based data analysis using CHIC is also performed based on a geometrical grid.

Gate template: Represents the entity of all gates. It is defined by marking all upcoming clusters of one defined experimental series and finally applied to all samples within this experiment.

Cytometric fingerprint (= cytometric pattern): It represents the microbial community structure by the number of clusters, the position of these clusters in the histogram, and the number of cells within each cluster.

Cytometric barcode (CyBar): Is the variation of the cytometric fingerprint over time or in dependence on experimental factors.

Operator dependence: The personal impact on the data evaluation procedure differs between methods, e.g., manual gating vs. automatic grid procedure. A method is defined as operator independent, if a meaningful result can be obtained by using predefined automatic settings. Usually, a cytometric background of the operator is not vital.

Cytometric Histogram Image Comparison (CHIC, Koch et al., 2013a), Cytometric Barcoding (CyBar, Koch et al., 2013b,c), and FlowFP (Rogers and Holyst, 2009; De Roy et al., 2012). The four methods differ in their analyzing principles and procedures. Therefore, this article will contrast and evaluate the four tools regarding methodical differences. Subsequently, they are assessed toward their ability to resolve variations in cytometric fingerprints. As example, a FCM biological data set resulting from eight electroactive microbial biofilms grown under different substrate and inoculum conditions and being characterized on their performance parameters is evaluated using all methods.

\section{METHODS}

\section{PRINCIPLES OF DATA ANALYSIS}

Four methods can be used to evaluate cytometric fingerprint data sets of microbial communities: Dalmatian Plot, CHIC, CyBar, and FlowFP. Their working principles and procedures are explained in the following and summarized in Figure 2.

\section{Dalmatian Plot}

The Dalmatian Plot was first described by Bombach et al. (2011). The name refers to the simplified black-and-white images that are generated during the analyzing procedure. The first step of the procedure is that the most abundant subsets of cells in the $2 \mathrm{D}$ histograms are encircled by the operator. This is performed for each plot, i.e., measurement, individually (the gating procedure).
The resulting images then represent black blots on a white background for every measurement. Noteworthy, the relative abundance information of the individual clusters is lost when only the black-and-white combination is used, thus representing presence/ absence information. Higher resolved information can be obtained if the cell number is integrated as gray level of the blots (Bombach et al., 2011; Müller et al., 2012). In the second step, the simplified images are processed with image analysis software. This image analysis procedure can be automatically performed using ready-to-use macros (http://www.ufz.de/index.php?en=32660) and the freely available software ImageJ (Schneider et al., 2012). The macros determine the relative area of the gates in each image by counting the number of black pixels. Then, an overlap image for each pair of images is created and the pixel number of overlapping gates determined. Afterwards, the dissimilarity $P_{\text {sim }}$ between each pair of images is calculated based on a modified Jaccard index or distance, respectively (Bombach et al., 2011; Patil et al., 2011). A dissimilarity matrix of all pairs of images under study is automatically created and can be used for statistical analysis and visualization in an ordination plot or cluster analysis, e.g., using $\mathrm{R}$ (R Core Team, 2012). All macros and a detailed description of the procedure are available under the QR-Code provided in Figure 2 and the following link http://www.ufz.de/index.php?en $=32660$.

\section{Cytometric Histogram Image Comparison (CHIC)}

CHIC is also an image based analysis tool but, in contrast to Dalmatian Plot, does not require an initial manual gating step. 


\section{Workflow}

I. Define area of events that represents DAPI stained cells.

II. Choose analyzing strategy from options a-d.

III. Visualize results in ordination plot.

A Dalmatian Plot
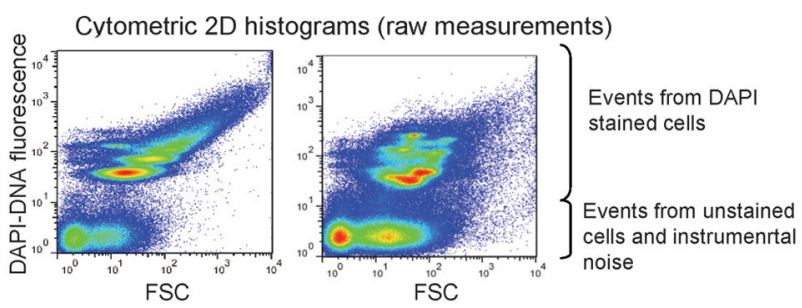

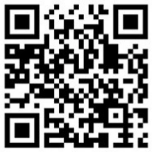

1) Prepare images representing the cell clusters for each sample.

Classically, the gates are represented as black schemes. Cell abundance information can be included by manually colouring the gates in gray scales.

2) Apply ImageJ macros to determine overlap area.

3) Calculate dissimilarity matrix with $\mathrm{R}$ macro.

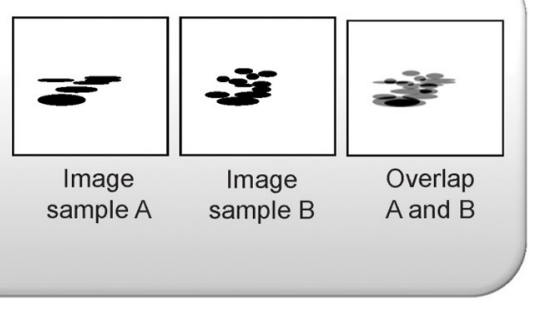

\section{B $\mathrm{CHIC}$}

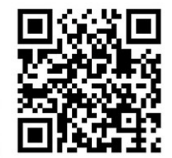

1) Prepare grey scale images from cytometric raw measurements.

2) Apply Image J macros to create overlap and $X O R$ image and determine the average grey value of informative pixel in XOR image.

3) Calculate dissimilarity matrix with $R$ macro.

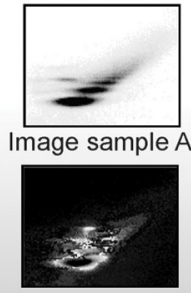

XOR $A$ and $B$
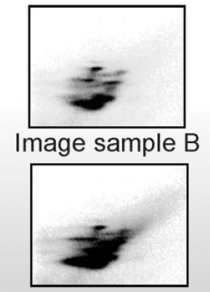

Overlap A and B

\section{CyBar}

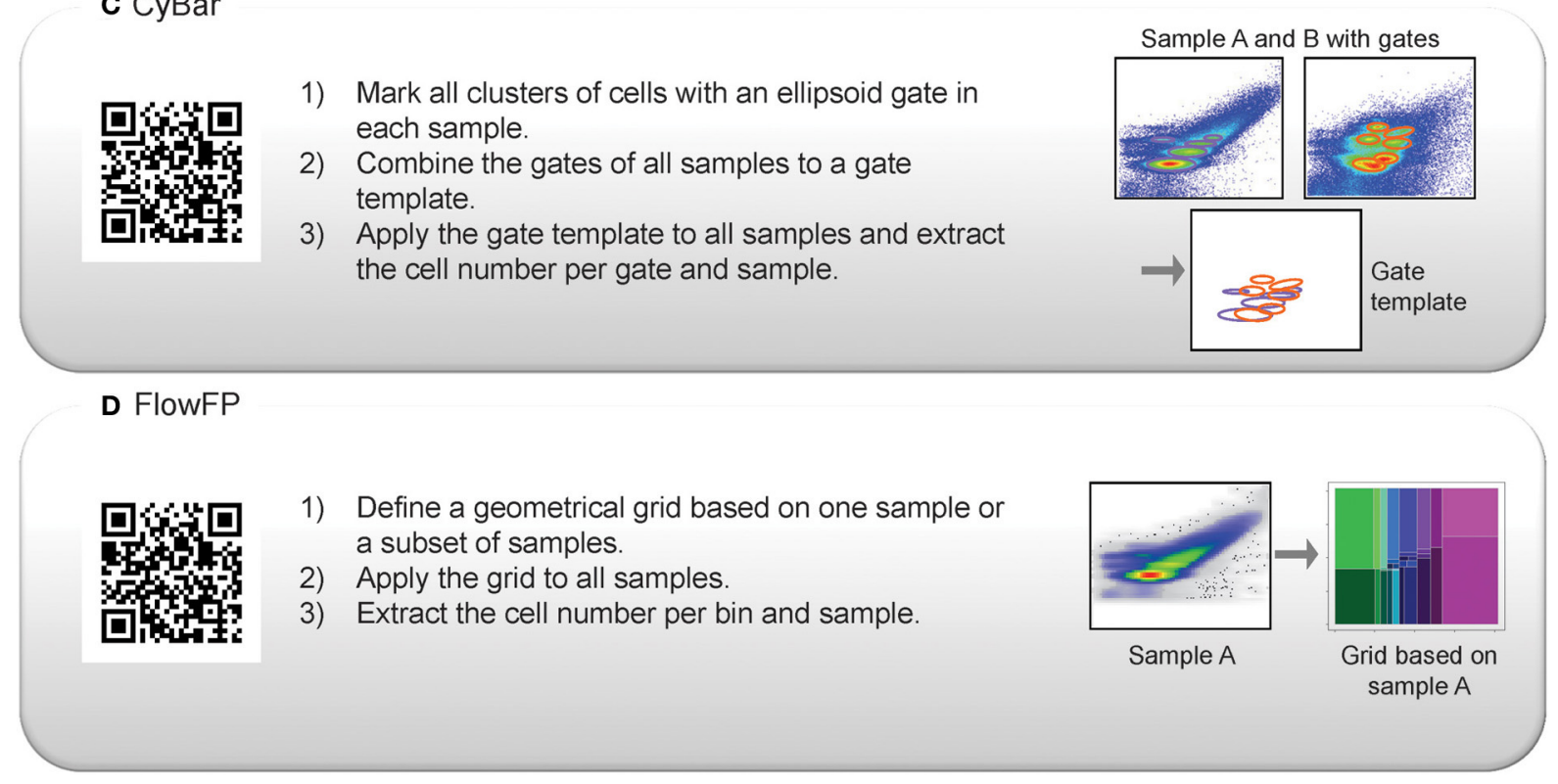

FIGURE 2 | Workflow for the data analysis procedure of cytometric fingerprints applying Dalmatian Plot, CHIC, CyBar, or FlowFP. The QR code links to the ready-to-use files for each procedure.

The $2 \mathrm{D}$ histograms are directly converted to gray scaled images using cytometric software like Summit 3.1 (DakoCytomation). Based on the binary code of the electronic signal acquisition, a histogram resolution in the range of $64,128,256,512$, or 1024 channel numbers can usually be chosen and will be reflected in images that will be created from the histograms. Each image will, therefore, have a defined resolution, representing the size of a grid. To evaluate the images the same regular grid should be chosen 
for all measured samples of a sampling campaign. Images are created from histograms that represent the virtual cells in a linear gray scale intensity representative for the relative abundance of cells per grid. Using ImageJ the cytometric images are compared to each other. This comparison is based on two mathematical computations, which are performed on a pixel to pixel basis for every pair of images. The exclusive disjunction function creates an XOR image of two cytometric images while the second algorithm produces their overlap. Subsequently, the average gray value per informative pixel, i.e., pixel resulting from virtual cells, is calculated using the sum of all pixel values from the XOR image and the number of informative pixels from the overlap image. The average gray value can directly be used as dissimilarity value $P_{\text {sim }}$ for each pair of images and a dissimilarity matrix of all pairs of images is automatically created. It can be used in the same way as with Dalmatian Plot for statistical analysis and visualization. All macros are available in (Koch et al., 2013a) and can also be found under the QR-Code provided in Figure 2 and the following link http://www.ufz.de/index.php?en=32736.

\section{Cytometric Barcoding (CyBar)}

CyBar performs a segregated analysis of cytometric histograms without any image analysis step. In this procedure, like in most analyzing procedures in FCM, an operator dependent, and thus experience based gating step has to be performed. Every cluster of cells in a histogram is marked with a gate. The individual gates of each sample are combined to one gate template for a data set. Such a gate template can comprise up to 30 gates and more when natural microbial community data sets are explored (Koch et al., 2013c). The gate template serves then as a mask which is applied to all samples of the data set. The cell abundances in each gate are easily extracted for all samples. Therewith, the abundance variation per gate can directly be compared between samples of different treatments or over a time course. The direct comparison of cell abundance variations between gates with high and low cell numbers is facilitated by data normalization. The dynamic variations of abundances per gate are then visualized in form of a barcode like heat map, the CyBar plot (performed in R, Figure S1). The CyBar plot allows identifying stable or highly fluctuating subsets of cells. In this way, a segregated analysis of individual cell cluster responses is possible in addition to the general trend interpretation analysis which was already provided by Dalmatian Plot and CHIC. Moreover, index subcommunities can be identified, i.e., potential functions assigned to clusters of cells by correlation analysis, and sorted for further analysis. A detailed step by step procedure and ready-to-use macros for the CyBar procedure are provided in Koch et al. (2013c) and were recently published as R package on the Bioconductor platform (www.bioconductor. com as flowCyBar, http://www.bioconductor.org/packages/devel/ bioc/html/flowCyBar.html). The link is also available under the QR-Code provided in Figure 2.

\section{FlowFP}

FlowFP (Rogers and Holyst, 2009) is a software package of the Bioconductor platform (Gentleman et al., 2004). Thereby, the complete analyzing procedure can be performed in R. FlowFP was first developed for handling FCM data sets for medical research, but was recently also successfully applied to a microbiological data set (De Roy et al., 2012). The FlowFP analyzing procedure does not require an image analysis step or any manual gating decision but works on the basis of a geometrical grid. The application uses a probability distribution function to define two regions of the FCM histogram that contain an equal number of cells. These regions are considered as bins and further partitioned with the identical probability distribution function creating equal sub-bins with identical virtual cell numbers. This procedure is repeated for every bin, based on a predefined number of recursions. The result is a geometrical grid with fixed numbers and positions of bins. Consequently, bins in regions with high abundance of virtual cells are smaller compared to those covering regions with low cell abundance. The grid can be built based on one sample or a set of samples. Subsequently, the computed grid serves as a mask which is applied to a complete data set. The number of cells per bin is extracted and stable and fluctuating bins can be identified. Therewith, segregated dynamics within microbial communities can be investigated as well as similarity analyses performed. For extensive information on FlowFP see Rogers and Holyst (2009) and (http://www.bioconductor.org/ packages/release/bioc/html/flowFP.html). A ready-to-use macro for the application of FlowFP to microbial cytometric fingerprints based on FSC and DAPI-DNA fluorescence (application example below) was created and is available under the QR-Code provided in Figure 2 and the following link http://www.ufz.de/index.php?en=32738.

\section{EXPERIMENTAL SETUP OF APPLICATION EXAMPLE}

The applicability of the above described four approaches for FCM data analyses was tested with a real biological data set. Anodic mixed culture derived electroactive microbial biofilms were characterized electrochemically and using FCM. Two important variables for the formation and performance of electroactive microbial biofilms were studied: (i) the inoculum, i.e., the source of the bacterial diversity, and (ii) the microbial substrate, i.e., the electron donor and carbon source. The outcome gained from the FCM data analysis was further evaluated using productivity parameters, i.e., biomass formation, maximum geometric current density $\left(j_{\max }\right)$, and coulombic efficiency $(C E)$.

\section{Electrochemical measurements}

All electrochemical experiments were carried out under potentiostatic control, using one-chamber three-necked-flasks $(250 \mathrm{~mL})$ with a three electrode arrangement consisting of the working electrode (projected surface area: $8.00 \mathrm{~cm}^{2}$ ), $\mathrm{Ag} / \mathrm{AgCl}$ reference electrode (saturated KCl, Sensortechnik Meinsberg, Germany, $0.195 \mathrm{~V}$ vs. SHE), and counter electrode. The working and counter electrodes used throughout this study were graphite rods (CP-Graphite $\mathrm{GmbH}$, Germany). The experiments were conducted with a Potentiostat/Galvanostat Model VMP3 (BioLogic Science Instruments, France), equipped with 12 independent potentiostat channels. The current density $\left(j_{\max }\right)$ is reported per projected surface area and denominated as "geometric current density." All experiments were conducted under anoxic conditions at $35^{\circ} \mathrm{C}$. 
Four types of wastewater served as microbial inoculum, i.e., primary wastewater (PW), activated sludge (AS), primary sludge (PS), and secondary sludge (SS). The wastewater samples were collected from the wastewater treatment plant Steinhof, Braunschweig (Germany). The growth medium was prepared as reported by Kim et al. (2005). In order to ensure anaerobic conditions it was purged with nitrogen for $30 \mathrm{~min}$ before use. Sodium acetate $(10 \mathrm{mM})$ or sodium lactate $(10 \mathrm{mM})$ served as substrates in the growth medium. An overview on sample denomination, source of microbial inoculum, and substrate choice is given in Table 1 .

The biofilm formation procedure was followed as described by Liu et al. (2008) in fed-batch experiments. $200 \mathrm{~mL}$ of the stirred growth medium were inoculated with $10 \mathrm{~mL}$ microbial inoculum. A constant potential of $+0.2 \mathrm{~V}$ (vs. $\mathrm{Ag} / \mathrm{AgCl}$ ) was applied to the working electrode to facilitate the biofilm formation. The biofilm growth was monitored by measuring the bioelectrocatalytic oxidation current. After substrate exhaustion (determined by HPLC and decrease in oxidation current) the bacterial medium was replenished using fresh solution.

Cyclic voltammetry $(\mathrm{CV})$ was performed during turnover conditions in accordance with previous studies, e.g., Fricke et al. (2008), Srikanth et al. (2008). Potentials were applied from -500 to $+300 \mathrm{mV}$ (vs. $\mathrm{Ag} / \mathrm{AgCl}$ ) at a scan rate of $1 \mathrm{mV} \mathrm{s}^{-1}$ with continuous monitoring of the current response (Fricke et al., 2008; Srikanth et al., 2008). The total coulombic efficiency (CE) was calculated by integrating the current over time and using the acetate respectively lactate consumption data. The electrochemical experiments were carried out three times and the biofilms of the third performance used for cytometric analysis. Original data are shown in Table S1.

\section{Analysis of chemical data}

The substrate consumption was assessed using HPLC. The HPLC (Spectrasystem P400, FINNIGAN Surveyor RI Plus detector, Fisher Scientific, Germany) was equipped with a Rezex HyperREZ $\mathrm{XP}$ Carbohydrate $\mathrm{H}+8 \mu \mathrm{m}$ column. The chromatograms were recorded at room temperature with $0.005 \mathrm{~N}$ sulphuric acid as eluent. Biomass was determined in triplicates as dry weight as described in Patil et al. (2011).

\section{Flow cytometry}

The biofilm samples were fixed with $10 \%$ sodium azide and prepared for cytometric analysis as described in Patil et al. (2011).

Table 1 | Overview on source of microbial inoculum and substrate for mixed culture derived microbial biofilm experiments and derived sample denomination.

\begin{tabular}{llc}
\hline Source of inoculum & Substrate & Sample denomination \\
\hline Primary wastewater (PW) & Acetate & $1 \mathrm{~A}$ \\
Primary wastewater (PW) & Lactate & $1 \mathrm{~L}$ \\
Activated sludge (AS) & Acetate & $2 \mathrm{~A}$ \\
Activated sludge (AS) & Lactate & $2 \mathrm{~L}$ \\
Primary sludge (PS) & Acetate & $3 \mathrm{~A}$ \\
Primary sludge (PS) & Lactate & $3 \mathrm{~L}$ \\
Secondary sludge (SS) & Acetate & $4 \mathrm{~A}$ \\
Secondary sludge (SS) & Lactate & $4 \mathrm{~L}$
\end{tabular}

The sample preparation included washing steps to remove the fixative, separation of the cells by vortex and sonication, and staining using a two-step procedure. The first step is $20 \mathrm{~min}$ incubation with a solution containing citric acid and Tween 20 to facilitate dye penetration and binding. Afterwards, the samples are incubated with the DAPI staining solution $(0.68 \mu \mathrm{M})$ for at least $60 \mathrm{~min}$.

The flow cytometric measurements were carried out as described before Patil et al. (2011). A MoFlo cell sorter (DakoCytomation, USA) equipped with two lasers [488 nm and ML-UV (333-365 nm)] was used to analyze FSC, SSC (trigger signal), and DAPI-DNA fluorescence. Fluorescent beads were used to align the instrument: yellow-green fluorescent microspheres $(2 \mu \mathrm{m}$, FluoSpheres (505/515), Molecular Probes, cat. no. F-8827), blue fluorescent microspheres $(1 \mu \mathrm{m}$, FluoSpheres (350/440), Molecular Probes, cat. no. F-8815), bright blue Fluoresbrite carboxylate microspheres $(0.5 \mu \mathrm{m}(360 / 407)$, Polysciences, cat. no. 18339-10). Data acquisition was performed with the Summit v.4.3 software (DakoCytomation, USA).

\section{Data analysis procedure}

The cytometric measurements of the obtained eight biofilms were analyzed with Dalmatian Plot, CHIC, CyBar, and FlowFP following the above described standard procedures and using the provided macros.

For the Dalmatian Plot the cytometric measurements were converted to simplified black-and-white images which represented the cytometric fingerprint with 5-11 black gates in each image, thus giving equal priority to all emerging clusters independent of their cell abundance. CHIC analysis was performed using a 128 channel resolution and the provided gray scale of the standard procedure. For CyBar, a gate template was constructed consisting of 20 gates. The FlowFP analysis was performed using the samples $1 \mathrm{~A}$ and $1 \mathrm{~L}$ to compute the grid based on 5 recursions.

\section{Statistics}

Statistical analysis was performed with $\mathrm{R}$ using the functions "metaMDS," "envfit," and "procrustes" from the package vegan (Oksanen et al., 2012). Correlation analysis was based on 999 permutations.

\section{RESULTS AND DISCUSSION COMPARISON OF TOOLS FOR MICROBIAL COMMUNITY ANALYSIS}

The four methods Dalmatian Plot, CHIC, CyBar, and Flow FP are available to follow variations in microbial community structures based on cytometric fingerprinting (Figure 2). Here, all four were applied to analyze the same biological data set resulting from eight electroactive microbial biofilms. First, the four tools are assessed regarding methodical differences (see also Table 2). Then, they are compared toward their ability to resolve specific variations in the microbial community structures and interpret community behavior in general and biofilm performance in particular.

\section{Impact of operator}

The four methods require different operator expertise with cytometric fingerprint analysis. So far, none of the analysis protocols can be used as "one-click-method."

The Dalmatian Plot and CyBar procedure require gating decisions by the operator at the beginning of the analyzing procedure. 
Table 2 | Comparison of the cytometric fingerprint evaluation tools.

\begin{tabular}{|c|c|c|c|c|}
\hline & Dalmation plot & CHIC & CyBar & FlowFP \\
\hline Outcome & Dissimilarity matrix & Dissimilarity matrix & $\begin{array}{l}\text { Matrix with cell numbers } \\
\text { per gate for all samples, } \\
\text { CyBar plot }\end{array}$ & $\begin{array}{l}\text { Matrix with cell numbers } \\
\text { per bin for all samples }\end{array}$ \\
\hline Software requirements & $\begin{array}{l}\text { Cytometric software, } \\
\text { Irfan-View, Paint, ImageJ, R }\end{array}$ & $\begin{array}{l}\text { Cytometric software, } \\
\text { ImageJ, R }\end{array}$ & Cytometric software, $\mathrm{R}$ & $\mathrm{R}$ \\
\hline Detection level of changes & Whole community & Whole community & Individual gate & Individual bin \\
\hline Advantages & $\begin{array}{l}\text { Simple, trend } \\
\text { interpretation analysis }\end{array}$ & $\begin{array}{l}\text { Operator independent, } \\
\text { fast, trend interpretation } \\
\text { analysis }\end{array}$ & $\begin{array}{l}\text { Segregated analysis of } \\
\text { subcommunity dynamics } \\
\text { in addition to trend } \\
\text { interpretation analysis, } \\
\text { matrix can be used for } \\
\text { subcommunity sorting }\end{array}$ & $\begin{array}{l}\text { Operator independent, } \\
\text { segregated analysis of } \\
\text { dynamics in bins in } \\
\text { addition to trend } \\
\text { interpretation analysis }\end{array}$ \\
\hline Disadvantage & $\begin{array}{l}\text { Experience based gating } \\
\text { procedure, time } \\
\text { consuming }\end{array}$ & $\begin{array}{l}\text { Conversion of histogram to } \\
\text { image }\end{array}$ & $\begin{array}{l}\text { Experience based gating } \\
\text { procedure }\end{array}$ & $\begin{array}{l}\text { Biological subcommunities } \\
\text { are not represented by } \\
\text { binning procedure }\end{array}$ \\
\hline
\end{tabular}

Gating decisions are individual and also strongly depend on the individual pre-experience with cytometric data analysis. For instance, while one operator defines a smaller number of bigger gates covering clusters of cells in a 2D histogram another operator will use a higher number but smaller gates analyzing the same data set. In general, the gates have to reflect the biological relevant clusters of cells in the histograms and should cover the majority of all virtual cells to allow reasonable interpretation. Histogram visualization using different graph types like pseudocolor, contour, or density plot helps to identify these clusters of cells.

The CHIC procedure is performed without any gating decisions as the complete histogram is converted to an image file. Therewith, the analysis procedure with CHIC is not operator dependent and always leads to the same result matrix. Nevertheless, the CHIC procedure includes adjustment options like the histogram resolution (Koch et al., 2013a). With these adjustments the sensitivity of the analysis can be improved to meet different requirements depending, e.g., on the individual experimental settings, number of recorded cells, or diversity of the microbial community. The FlowFP procedure also works without individual gating decisions, thus, could be performed without any operator impact. Nevertheless, the FlowFP procedure requires the computation of the grid that serves as mask to analyze the data. The definition of the grid is very flexible and certainly influences the analysis result. The sample choice for defining the grid should reflect the diversity of patterns to be analyzed in a data set. By that and an adequate number of recursions it is ensured that regions of high and low virtual cell density are equally covered in all samples and changes in the community structure can reliably be detected.

\section{Detection level of differences}

All four approaches result in a mathematical matrix, which finally allows visualizing dissimilarities between samples and thus structures of microbial communities.

For Dalmatian Plot and CHIC this matrix represents a dissimilarity matrix which results from the pair wise comparison of all histograms. Each comparison results in a dissimilarity value between two given samples. The value represents the overall dissimilarity for each pair and does not enable to retrieve information about the source of dissimilarity. The dissimilarity can either result from only one cluster of cells showing a strong change in abundance between two samples or from several smaller changes in more than one cluster. However, it is often of high interest to know details about the changes in the microbial community structure. Segregated analysis of subcommunity dynamics in addition to trend interpretation analysis is only possible with CyBar and FlowFP. Both enable the detection of structural community changes down to the individual gate or bin, respectively.

For CyBar the gate template based analysis allows directly visualizing the differences in abundance of each subset of virtual cells using the CyBar plot (see also Figure S1). Therewith, it is easily possible to allocate changes in community structure to certain clusters that rise or reduce their cell numbers. In addition, clusters with similar or opposite response can be identified. The gate template has another advantage. It can directly be used as template for cell sorting; thus, cell clusters of interest can be manually separated from the whole community and afterwards further investigated based on genomic or proteomic techniques (Jahn et al., 2013; Koch et al., 2013c).

FlowFP also allows identifying bins which are stable or show a high variability. Due to the principle of probability binning with equal numbers of cells per bin, regions of very high cell density will result in several smaller rectangular bins in the two dimensional plot. Thus, changes will most often not be restricted to one single bin but affect a number of neighboring bins. However, a bin is an artificial classification based on cell number distribution and independent of potential biological subcommunities. Therefore, it does not mark biological subsets of cells making cell sorting and cell cluster based interpretation, e.g., regarding proliferation changes, more difficult.

As a result of the individual detection levels of differences, the interpretation depth is also limited dependent on the chosen analysis. The structural changes in the microbial community are a response to changes in environmental or experimental 
parameters. Using statistical analysis the overall change between samples can be correlated with the experimental variables. This general trend interpretation is possible for all four techniques. In addition, the segregated analysis of CyBar and Flow FP allows individual gate or bin correlations, thus the identification of functional subcommunities.

\section{APPLICATION EXAMPLE: CYTOMETRIC COMMUNITY ANALYSIS OF ELECTROACTIVE MICROBIAL BIOFILMS}

The electroactive microbial biofilms were formed at solid carbon electrodes which served as anode, i.e., electron acceptor, using a standard bioelectrochemical three electrode setup. The potential at the anode was constant at $0.2 \mathrm{~V}$ (vs. $\mathrm{Ag} / \mathrm{AgCl}$ ). Microorganisms performing extracellular electron transfer (EET), i.e., possessing the ability to transfer electrons to solid terminal acceptors, can oxidize the provided substrates (acetate or lactate) to $\mathrm{CO}_{2}$ and $\mathrm{H}^{+}$. The resulting EET is measured as flow of electric current. The microbial community structure of the electroactive biofilms was determined using cytometric fingerprinting as method of choice (vide supra) and its performance characterized by electrochemical and biological parameters. Here, the following parameters were used (i) the maximum geometric current density, $j_{\text {max }}$, which is the maximum number of transferred electrons per second per projected surface area of the electrode, (ii) the Coulombic efficiency, $C E$, which is a measure of the electron transfer to the electrode per electron released by substrate oxidation reaction, and (iii) the biomass of the biofilm. For more details on electroactive microbial communities and their technological potential, e.g., Harnisch and Schröder (2010); Logan and Rabaey (2012); Rabaey and Rozendal (2010). In the current experiment two important variables for their formation and performance were studied: the types of inoculum and substrate.

The inoculum determines the microbial diversity, i.e., the present species, in each setup that will have the chance to colonize the electrode. The inoculum samples were collected from four basins of a local wastewater treatment plant (PW, AS, PS, SS—-see Table 1). As such basins provide different environmental conditions (e.g., carbon sources, oxygen levels, flow through rates), they are generally known to contain different microbial communities (Günther et al., 2012). The choice of substrate, i.e., the electron donor, determines the growth activity of those microorganisms in a bioelectrochemical cell, being able to utilize the substrate either directly by EET or indirectly as part of a food web, e.g., by fermentation. Therewith, the biofilm structure at the anode results from an electrochemically driven selection. Information on the structure of a microbial community in an electroactive biofilm may bare the potential to understand and predict its performance parameters. Therefore, the electroactive microbial biofilms were investigated using cytometric fingerprinting. The obtained histograms were analyzed with Dalmatian Plot, CHIC, CyBar, and Flow FP and the results visualized using non-metric multidimensional scaling (NMDS) plots (Figure 3). The FCM results were furthermore correlated to the bulk measurements of performance parameters in order to reveal structure-function relationships of biofilm productivity and microbial community (see Günther et al., 2012, for comparison).
The results of the dissimilarity analyses of the microbial biofilms were comparable for all four methods. From all plots in Figure 3 it is evident that the origin of the bacterial community, i.e., the source of inoculum, had a major influence on the community structure of the biofilms. The biofilms resulting from the same inoculum were more similar to each other based on their cytometric fingerprints although different substrates were provided. In addition, it has to be mentioned that the cytometric fingerprints of the biofilms were differing from those of the microbial inocula (Figure 1 and Figure S2) emphasizing the electrochemically driven selection. The substrate sources, acetate and lactate, were also found to cause differences in the microbial community structures between the biofilms, but the inoculum was the major determinate.

Two out of eight biofilms, $1 \mathrm{~A}$ and $1 \mathrm{~L}$, with the origin from primary wastewater showed a high electrochemical performance in terms of $j_{\max }$ and $C E$ (for comparison, e.g., Harnisch and Schröder, 2010; Pant et al., 2010). They reached the highest current densities of $659 \mu \mathrm{A} \mathrm{cm} \mathrm{cm}^{-2}$ and $494 \mu \mathrm{A} \mathrm{cm} \mathrm{cm}^{-2}$, and the highest Coloumbic efficiencies of $94 \%$ and $25 \%$, respectively, accompanied by high biomass formation [3.68 $( \pm 0.76)$ and 4.17 $\left.( \pm 0.28) \mathrm{mg} \mathrm{cm}^{-2}\right]$. Both biofilms showed a comparable maximum performance, but only the biofilm $1 \mathrm{~A}$ used $94 \%$ of the consumed substrate (acetate) for the current production. This is in line with literature, as high performing electroactive microorganisms like Geobacter can directly utilize acetate, but not lactate (Speers and Reguera, 2012). In former studies, high performing biofilms were characterized on their electrochemical behavior and possessed properties similar to previous mixed culture derived biofilms that were dominated by Geobacteraceae (Torres et al., 2009; Harnisch et al., 2011; Patil et al., 2011). The second best performing pair of biofilms was $2 \mathrm{~A}$ and $2 \mathrm{~L}$ resulting from the activated sludge inoculum. Their cytometric fingerprints showed a higher similarity to $1 \mathrm{~A}$ and $1 \mathrm{~L}$ than the other biofilms, which was also reflected by the plots in Figure 3. The more the cytometric fingerprints of the biofilms deviated from $1 \mathrm{~A}$ and $1 \mathrm{~L}$ the less bioelectrochemically productive were they, along with lower biomass formation $\left[1.21( \pm 0.14)\right.$ to $\left.2.50( \pm 1.09) \mathrm{mg} \mathrm{cm}^{-2}\right]$. This interrelationship between the origin of the community, the resulting biofilm formation, and its productivity can clearly be retrieved from the cytometric data sets and is emphasized by correlation analysis (Figure 3).

From a biological perspective, the microorganisms with the highest capacity for establishing electroactive microbial biofilms seemed preferentially present in the primary wastewater. Primary wastewater is a highly variable habitat which is characterized by numerous organic substances as it is the first basin in the wastewater treatment flow that contains high amounts of C-and $\mathrm{N}$-species. In contrast to the various wastewater basins, the experimental setup offered a highly specialized niche. As expected, the originally highly diverse structure of the primary wastewater community decreased and only a small number of specialists formed the electroactive biofilm. The structural and functional differences of the biofilms in the different experimental setups were well recognized by all four tools (Figure 3 and discussion below) and could be used as basis for further ecological interpretation (Box 2). From an engineering perspective, these results 


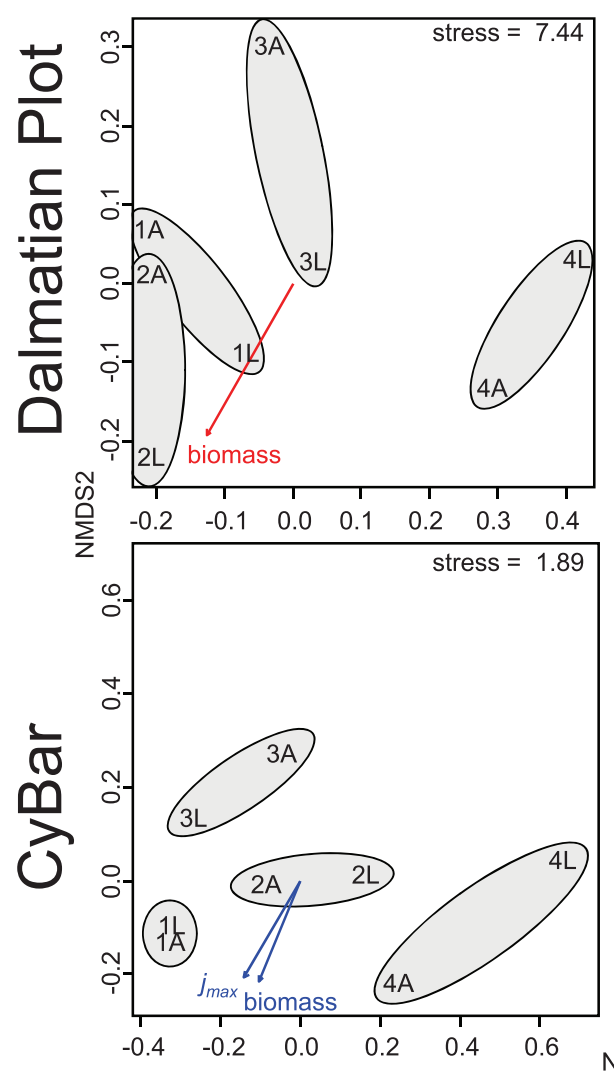

FIGURE 3 | Dissimilarity analysis results of cytometric fingerprints of electroactive biofilms. The cytometric fingerprints of eight biofilms were recorded and analyzed with Dalmatian Plot, CHIC, CyBar, and FlowFP. The samples are arranged according to their dissimilarity in the NMDS plots and the gray circles indicate samples with the same

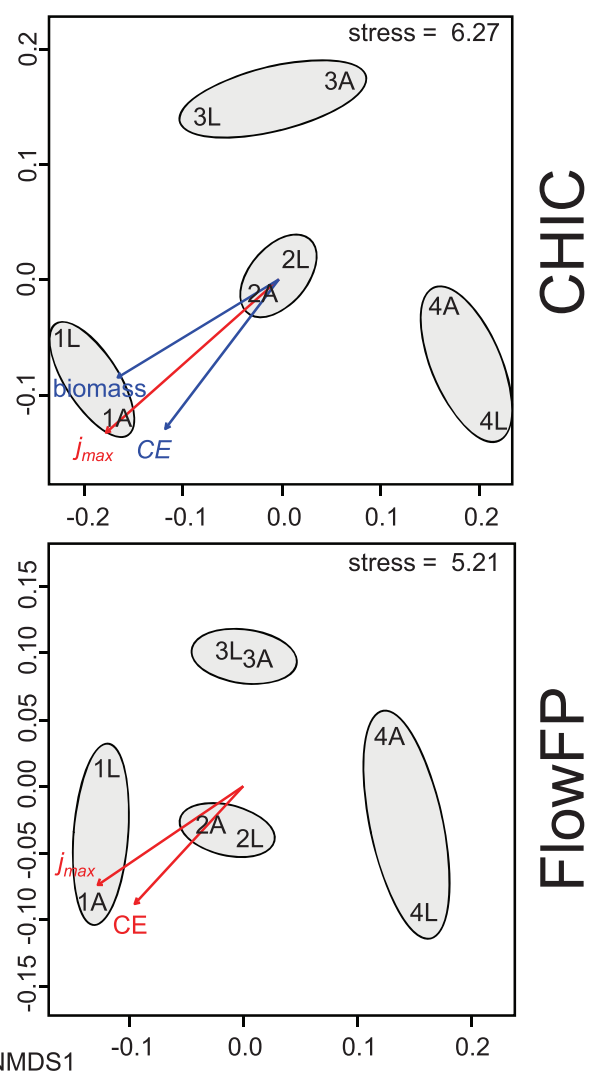

inoculum. For further sample denomination see Table 1. The productivity parameters maximum geometric current density $\left(j_{\max }\right)$, coulombic efficiency (CE), and biomass of the biofilm were correlated to the cytometric results and significant correlations are displayed with $p=0.05$ in red and $p=0.1$ in blue. can be of highest value for planning and implementation of bioelectrochemical setups into wastewater treatment plants (Lovley, 2006; Rabaey et al., 2010). The high costs and risks for empirical testing can be reduced using FCM in combination with correlation analysis beforehand as exemplarily demonstrated in the application example.

\section{COMPARABILITY OF RESULTS}

The dissimilarity analysis results of the application example show a high resemblance for all four tools (Figure 3). CyBar, FlowFP, and CHIC show highest similarity in the NMDS plots, which was also supported by procrustes analysis (Table S2), a mathematical procedure to compare ordination results. Possible reasons for the higher deviation of the Dalmatian Plot are (i) that this method is based on individual gating per sample in contrast to the global template (gate, respectively grid), used for the other tools, as well as (ii) that no cell abundance information is exploited (by only using the black-and-white, i.e., presence-absence information).

The correlation of FCM data with the productivity parameters using all four tools showed similar dependencies. Commonly, biofilms derived from primary wastewater and activated sludge resulted in the best and second best performance regarding biomass, $j_{\text {max }}$, and CE. Nevertheless, individual differences on the methods were found. Whereas Dalmatian Plot identified only biomass as significant correlation parameter $(p=0.05)$ two significant correlation parameters were found with CyBar $\left(j_{\max }\right.$ and biomass, $p=0.1$ ) and FlowFP ( $j_{\max }$ and CE, $\left.p=0.05\right)$. With CHIC significant correlations were found for $j_{\max }(p=0.05)$, biomass, and CE $(p=0.1)$.

The major focus for the application example was the detection of trends in community structure variation. This was achieved by using all tools. CHIC and FlowFP offered the fastest analyzing procedures for this purpose including high reproducibility. If a further segregated analysis of subcommunity dynamics, i.e., the contribution of individual subcommunities to the overall change, is aimed, CyBar provided the best option (Figure S1).

\section{FROM THE DETECTION OF CHANGES TO BIODIVERSITY INDICES}

New cytometric fingerprinting methods allow changes in microbial community structures to be followed. Independent of the different motivations for monitoring microbial communities different information can be derived. One of the prime options is monitoring community stability, which is, e.g., needed for 


\section{Box 2 | Ecological interpretation of cytometric data sets.}

In macro- as well as in microbiology biodiversity indices help to investigate large data sets derived from community analysis on respective community characteristics and response to environmental changes (Purvis and Hector, 2000; Prosser et al., 2007; Marzorati et al., 2008). However, specific values for these indices defining a community, e.g., as divers, healthy, or functional are missing (Purvis and Hector, 2000; Read et al., 2011). As demonstrated in the following on the example of CHIC respective cytometric diversity indices can be derived from the cytometric fingerprint. Yet, further comprehensive data set analyses are needed for benchmarking them.

Range-weighted richness $(R r)$

The $R r$ value represents the number of detected distinct individuals, $N_{i}$, in relation to the total number of distinct individuals, $N_{\text {all }}$. As in FCM individuals are represented by virtual cells in histogram $\mathrm{Rr}$ can be calculated from the cut images (see $\mathrm{CHIC}$ procedure) according to equation 1:

$$
\operatorname{Rr}=\frac{N_{i}}{N_{a l l}}
$$

with $N_{i}$ being the number of pixels containing virtual cell information (being not white) and $N_{\text {all }}$ the number of all pixels of the cytometric image (including informative and non-informative, thus white, pixels).

Structural organization (So)

So describes the relative difference in abundance of distinct individuals, $P_{i j}$, and the average abundance of all distinct individuals, $P_{a v e r a g e}$ (see Marzorati et al., 2008). Thus, in cytometric fingerprints the single pixel value represents $P_{i j}$ and the average pixel intensity of all informative pixels represents $P_{\text {average }}$ as follows:

$$
\text { So }=\frac{\sum_{i ; j}\left|P_{i j}-P_{\text {average }}\right|}{N_{i}}
$$

with $\mathrm{Ni}$ being the number of pixel bearing virtual cell information (being not white).

Dynamics $(D y, D a)$

Dynamics describe the degree of variation within a microbial community over time. Dy describes the average dissimilarity of consecutive samples and $\mathrm{Da}$ the average dissimilarities of all samples. In FCM all pairwise dissimilarity values are given in the $\mathrm{CHIC}$ result matrix (see Methods as well as Koch et al., 2013a) and can be used to calculate both indices.

\section{Application example}

The above described cytometric diversity indices $R r$ and So were calculated from the cytometric fingerprint data set of the application example. As the dynamic development of the biofilm was not monitored Da and Dy were not assessed.

The experimental setup offered a narrow ecological niche as only a single substrate (electron donor) and an electrode as solid electron acceptor were provided. As only a small number of microbial specialists can occupy this niche a reduced number of different individuals were expected. Consequently, these specialists will increase their numbers stronger than other individuals which are less adapted. This expected adaptation is reflected by the cytometric fingerprints of the biofilms. The highest specialization is detected in the PW derived biofilms grown on acetate and lactate, as represented by the lowest range-weighted richness $[R r=0.43(1 \mathrm{~A})$ and $R r=0.46(1 \mathrm{~L})]$ and high structural organization $[S o=39.3(1 \mathrm{~A})$ and $S o=43.0(1 \mathrm{~L})]$. These biofilms were also the most productive ones in terms of current production and efficiency (see Table S1). For results of all biofilms see Table S3.

the evaluation of drinking water quality (De Roy et al., 2012; Hammes et al., 2012; Prest et al., 2013). Here, a continuous and fast analyzing procedure is required allowing an instantaneous statement on the (in)stability. As was shown in the current study, CHIC and FlowFP are best suited tools for analyzing FCM-data sets for this kind of application. A more detailed insight into the community changes is given with the second option, the segregated analysis of microbial subcommunity changes. It allows identifying individual contributions of subcommunities to an overall process as, e.g., found in the different basins of a wastewater treatment plant (Günther et al., 2012). Here, CyBar is the best choice for the cytometric fingerprint analysis.
Furthermore, biodiversity measures, which are well established in macroecology theory and that were already applied to taxonomy based microbial data sets (e.g., Purvis and Hector, 2000; Prosser et al., 2007; Marzorati et al., 2008), can also be derived from the cytometric fingerprints. This has been predicted by Wang et al. (2010) but until now no mathematical implementation was shown. Box 2 gives a first approach on how the cytometric diversity indices (i) range-weighted richness, (ii) structural organization, and (iii) dynamics of microbial communities can be calculated from cytometric fingerprints. Henceforth, these indices can help to derive further community characteristics and investigate underlying ecological principles for microbial community behavior. 


\section{CONCLUSION}

All four analysis tools (CHIC, Dalmatian Plot, CyBar, and FlowFP) allow deriving further information of FCM fingerprints. As the tools differ in their principles of data analysis, their detection level of changes differs as well. As FCM fingerprints can sensitively resolve dynamics in microbial communities with high throughput and at low costs, their application for monitoring managed microbial systems (e.g., biotechnology, energy production, drinking water supply) as well as natural environments is highly recommended. Thereby, microbial FCM can be flexibly expanded with advanced -omics techniques in scientific research as well as with practical implementations (Koch et al., 2014a,b).

\section{ACKNOWLEDGMENTS}

We thank A. A. Carmona-Martinez, Sunil A. Patil, SiangFu Hong, T. Hübschmann, and C. Süring for technical assistance and M. Jahn for introduction to Bioconductor and FlowFP. Falk Harnisch acknowledges support by the BMBF (Research Award "Next generation biotechnological processes-Biotechnology 2020+") and the Helmholtz-Association (Young Investigators Group). Susann Müller acknowledges support by the European Fond for Regional Development (EFRE) and Sächsische AufbauBank (SAB). This work was supported by the Helmholtz Association within the Research Programme Renewable Energies.

\section{SUPPLEMENTARY MATERIAL}

The Supplementary Material for this article can be found online at: http://www.frontiersin.org/journal/10.3389/fmicb. 2014.00273/abstract

\section{REFERENCES}

Bombach, P., Hübschmann, T., Fetzer, I., Kleinsteuber, S., Geyer, R., Harms, H., et al. (2011). "Resolution of natural microbial community dynamics by community fingerprinting, flow cytometry, and trend interpretation analysis," in High Resolution Microbial Single Cell Analytics, Vol. 124, eds S. Müller and T. Bley (Berlin; Heidelberg: Springer), 151-181.

De Roy, K., Clement, L., Thas, O., Wang, Y., and Boon, N. (2012). Flow cytometry for fast microbial community fingerprinting. Water Res. 46, 907-919. doi: 10.1016/j.watres.2011.11.076

Fricke, K., Harnisch, F., and Schröder, U. (2008). On the use of cyclic voltammetry for the study of anodic electron transfer in microbial fuel cells. Energy Environ. Sci. 1:144. doi: 10.1039/b802363h

Gentleman, R., Carey, V., Bates, D., Bolstad, B., Dettling, M., Dudoit, S., et al. (2004). Bioconductor: open software development for computational biology and bioinformatics. Genome Biol. 5:R80. doi: 10.1186/gb-2004-5-10-r80

Günther, S., Koch, C., Hübschmann, T., Röske, I., Müller, R. A., Bley, T., et al. (2012). Correlation of community dynamics and process parameters as a tool for the prediction of the stability of wastewater treatment. Environ. Sci. Technol. 46, 84-92. doi: 10.1021/es2010682

Günther, S., Trutnau, M., Kleinsteuber, S., Hause, G., Bley, T., Röske, I., et al. (2009). Dynamics of polyphosphate-accumulating bacteria in wastewater treatment plant microbial communities detected via DAPI $\left(4^{\prime}, 6^{\prime}\right.$-Diamidino2-Phenylindole) and Tetracycline labeling. Appl. Environ. Microbiol. 75, 2111-2121. doi: 10.1128/AEM.01540-08

Hammes, F., Broger, T., Weilenmann, H.-U., Vital, M., Helbing, J., Bosshart, U., et al. (2012). Development and laboratory-scale testing of a fully automated online flow cytometer for drinking water analysis. Cytometry 81A, 508-516. doi: 10.1002/cyto.a.22048

Harnisch, F., Koch, C., Patil, S. A., Hübschmann, T., Müller, S., and Schröder, U. (2011). Revealing the electrochemically driven selection in natural community derived microbial biofilms using flow-cytometry. Energy Environ. Sci. 4, 1265-1267. doi: 10.1039/c0ee00605j
Harnisch, F., and Schröder, U. (2010). From MFC to MXC: chemical and biological cathodes and their potential for microbial bioelectrochemical systems. Chem. Soc. Rev. 39, 4433-4448. doi: 10.1039/c003068f

Jahn, M., Seifert, J., von Bergen, M., Schmid, A., Bühler, B., and Müller, S. (2013). Subpopulation-proteomics in prokaryotic populations. Curr. Opin. Biotechnol. 24, 79-87. doi: 10.1016/j.copbio.2012.10.017

Kim, J. R., Min, B., and Logan, B. E. (2005). Evaluation of procedures to acclimate a microbial fuel cell for electricity production. Appl. Microbiol. Biotechnol. 68, 23-30. doi: 10.1007/s00253-004-1845-6

Kleinsteuber, S., Riis, V., Fetzer, I., Harms, H., and Müller, S. (2006). Population dynamics within a microbial consortium during growth on diesel fuel in saline environments. Appl. Environ. Microbiol. 72, 3531-3542. doi: 10.1128/AEM.72.5.3531-3542.2006

Koch, C., Fetzer, I., Harms, H., and Müller, S. (2013a). CHIC-an automated approach for the detection of dynamic variations in complex microbial communities. Cytometry A. 83, 561-567. doi: 10.1002/cyto.a.22286

Koch, C., Fetzer, I., Schmidt, T., Harms, H., and Müller, S. (2013b). Monitoring functions in managed microbial systems by cytometric bar coding. Environ. Sci. Technol. 47, 1753-1760. doi: 10.1021/es3041048

Koch, C., Günther, S., Desta, A. F., Hübschmann, T., and Müller, S. (2013c). Cytometric fingerprinting for analyzing microbial intracommunity structure variation and identifying subcommunity function. Nat. Protoc. 8, 190-202. doi: 10.1038/nprot.2012.149

Koch, C., Harms, H., and Müller, S. (2014a). Dynamics in the microbial cytomesingle cell analytics in natural systems. Curr. Opin. Biotechnol. 27, 134-141. doi: 10.1016/j.copbio.2014.01.011

Koch, C., Müller, S., Harms, H., and Harnisch, F. (2014b). Managing microbiomes in bioenergy production: from analysis to proactive steering. Curr. Opin. Biotechnol. 27, 65-72. doi: 10.1016/j.copbio.2013.11.006

Liu, Y., Harnisch, F., Fricke, K., Sietmann, R., and Schröder, U. (2008). Improvement of the anodic bioelectrocatalytic activity of mixed culture biofilms by a simple consecutive electrochemical selection procedure. Biosens. Bioelectron. 24, 1006-1011. doi: 10.1016/j.bios.2008.08.001

Logan, B. E., and Rabaey, K. (2012). Conversion of wastes into bioelectricity and chemicals using microbial electrochemical technologies. Science 337, 686-690. doi: $10.1126 /$ science. 1217412

Lovley, D. R. (2006). Microbial fuel cells: novel microbial physiologies and engineering approaches. Curr. Opin. Biotechnol. 17, 327-332. doi: 10.1016/j.copbio.2006.04.006

Marzorati, M., Wittebolle, L., Boon, N., Daffonchio, D., and Verstraete, W. (2008). How to get more out of molecular fingerprints: practical tools for microbial ecology. Environ. Microbiol. 10, 1571-1581. doi: 10.1111/j.14622920.2008.01572.x

Meistrich, M. L., Göhde, W., White, R. A., and Schumann, J. (1978). Resolution of X and Y spermatids by pulse cytophotometry. Nature 274, 821-823. doi: $10.1038 / 274821 \mathrm{a} 0$

Müller, S. (2007). Modes of cytometric bacterial DNA pattern: a tool for pursuing growth. Cell Prolif. 40, 621-639. doi: 10.1111/j.1365-2184.2007.00465.x

Müller, S., Hübschmann, T., Kleinsteuber, S., and Vogt, C. (2012). High resolution single cell analytics to follow microbial community dynamics in anaerobic ecosystems. Methods 57, 338-349. doi: 10.1016/j.ymeth.2012.04.001

Müller, S., and Nebe-von-Caron, G. (2010). Functional single-cell analyses: flow cytometry and cell sorting of microbial populations and communities. FEMS Microbiol. Rev. 34, 554-587. doi: 10.1111/j.1574-6976.2010.00214.x

Oksanen, J., Blanchet, F. G., Kindt, R., Legendre, P., Minchin, P. R., O’Hara, R. B., et al. (2012). Package "vegan". Available online at: http://vegan.r-forge. r-project.org/

Pant, D., Van Bogaert, G., Diels, L., and Vanbroekhoven, K. (2010). A review of the substrates used in microbial fuel cells (MFCs) for sustainable energy production. Bioresour. Technol. 101, 1533-1543. doi: 10.1016/j.biortech.2009.10.017

Patil, S. A., Harnisch, F., Koch, C., Hübschmann, T., Fetzer, I., CarmonaMartínez, A. A., et al. (2011). Electroactive mixed culture derived biofilms in microbial bioelectrochemical systems: the role of $\mathrm{pH}$ on biofilm formation, performance and composition. Bioresour. Technol. 102, 9683-9690. doi: 10.1016/j.biortech.2011.07.087

Prest, E. I., Hammes, F., Kötzsch, S., van Loosdrecht, M. C., and Vrouwenvelder, J. S. (2013). Monitoring microbiological changes in drinking water systems using a fast and reproducible flow cytometric method. Water Res. 47, 7131-7142. doi: 10.1016/j.watres.2013.07.051 
Prosser, J. I., Bohannan, B. J. M., Curtis, T. P., Ellis, R. J., Firestone, M. K., Green, J. L., et al. (2007). The role of ecological theory in microbial ecology. Nat. Rev. Microbiol. 5, 384-392. doi: 10.1038/nrmicro1643

Purvis, A., and Hector, A. (2000). Getting the measure of biodiversity. Nature 405, 212-219. doi: 10.1038/35012221

Rabaey, K., Angenent, L., Schröder, U., and Keller, J. (eds.). (2010). Bioelectrochemical Systems: From Extracellular Electron Transfer to Biotechnological Application. London; New York: IWA Publishing.

Rabaey, K., and Rozendal, R. A. (2010). Microbial electrosynthesis-revisiting the electrical route for microbial production. Nat. Rev. Microbiol. 8, 706-716. doi: $10.1038 /$ nrmicro 2422

R Core Team (2012). R: A Language and Environment for Statistical Computing. Vienna.

Read, S., Marzorati, M., Guimarães, B. C. M., and Boon, N. (2011). Microbial Resource Management revisited: successful parameters and new concepts. Appl. Microbiol. Biotechnol. 90, 861-871. doi: 10.1007/s00253-0113223-5

Rogers, W. T., and Holyst, H. A. (2009). FlowFP: a bioconductor package for fingerprinting flow cytometric data. Adv. Bioinformatics 2009:193947. doi: $10.1155 / 2009 / 193947$

Schneider, C. A., Rasband, W. S., and Eliceiri, K. W. (2012). NIH Image to ImageJ: 25 years of image analysis. Nat. Meth. 9, 671-675. doi: 10.1038/nmeth.2089

Shapiro, H. M. (2003). Practical Flow Cytometry. New York, NY: Wiley and Sons. doi: $10.1002 / 0471722731$

Speers, A. M., and Reguera, G. (2012). Electron donors supporting growth and electroactivity of Geobacter sulfurreducens anode biofilms. Appl. Environ. Microbiol. 78, 437-444. doi: 10.1128/AEM.06782-11
Srikanth, S., Marsili, E., Flickinger, M. C., and Bond, D. R. (2008). Electrochemical characterization of Geobacter sulfurreducens cells immobilized on graphite paper electrodes. Biotechnol. Bioeng. 99, 1065-1073. doi: 10.1002/bit.21671

Torres, C. I., Krajmalnik-Brown, R., Parameswaran, P., Marcus, A. K., Wanger, G., Gorby, Y. A., et al. (2009). Selecting anode-respiring bacteria based on anode potential: phylogenetic, electrochemical, and microscopic characterization. Environ. Sci. Technol. 43, 9519-9524. doi: 10.1021/es902165y

Wang, Y., Hammes, F., De Roy, K., Verstraete, W., and Boon, N. (2010). Past, present and future applications of flow cytometry in aquatic microbiology. Trends Biotechnol. 28, 416-424. doi: 10.1016/j.tibtech.2010.04.006

Conflict of Interest Statement: The authors declare that the research was conducted in the absence of any commercial or financial relationships that could be construed as a potential conflict of interest.

Received: 09 April 2014; accepted: 19 May 2014; published online: 04 June 2014. Citation: Koch C, Harnisch F, Schröder U and Müller S (2014) Cytometric fingerprints: evaluation of new tools for analyzing microbial community dynamics. Front. Microbiol. 5:273. doi: 10.3389/fmicb.2014.00273

This article was submitted to Systems Microbiology, a section of the journal Frontiers in Microbiology.

Copyright (c) 2014 Koch, Harnisch, Schröder and Müller. This is an open-access article distributed under the terms of the Creative Commons Attribution License (CC BY). The use, distribution or reproduction in other forums is permitted, provided the original author(s) or licensor are credited and that the original publication in this journal is cited, in accordance with accepted academic practice. No use, distribution or reproduction is permitted which does not comply with these terms. 